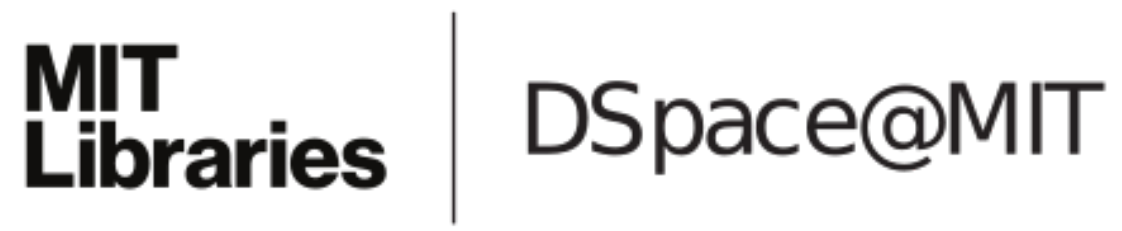

MIT Open Access Articles

Matching Points with Things

The MIT Faculty has made this article openly available. Please share how this access benefits you. Your story matters.

Citation: Aloupis, Greg et al. "Matching Points with Things." LATIN 2010: Theoretical Informatics. Ed. Alejandro López-Ortiz. Vol. 6034. Berlin, Heidelberg: Springer Berlin Heidelberg, 2010. 456467. Web. 25 June 2012.

As Published: http://dx.doi.org/ 10.1007/978-3-642-12200-2_40

Publisher: Springer Berlin / Heidelberg

Persistent URL: http://hdl.handle.net/1721.1/71205

Version: Author's final manuscript: final author's manuscript post peer review, without publisher's formatting or copy editing

Terms of use: Creative Commons Attribution-Noncommercial-Share Alike 3.0 


\title{
Matching Points with Things
}

\author{
Greg Aloupis ${ }^{1}$, Jean Cardinal $^{1}$, Sébastien Collette ${ }^{1 \star}$, Erik D. Demaine ${ }^{2}$, Martin \\ L. Demaine ${ }^{2}$, Muriel Dulieu ${ }^{3}$, Ruy Fabila-Monroy ${ }^{4}$, Vi Hart ${ }^{5}$, Ferran Hurtado ${ }^{6}$, \\ Stefan Langerman ${ }^{1 \star \star}$, Maria Saumell ${ }^{6}$, Carlos Seara ${ }^{6}$, and Perouz Taslakian ${ }^{1}$ \\ 1 Université Libre de Bruxelles, CP212, Bld. du Triomphe, 1050 Brussels, Belgium. \\ \{galoupis, jcardin, secollet, slanger, ptaslaki\}@ulb.ac.be. Supported by the \\ Communauté française de Belgique - ARC. \\ 2 MIT Computer Science and Artificial Intelligence Laboratory, 32 Vassar St., \\ Cambridge, MA 02139, USA. \{edemaine,mdemaine\}@mit.edu \\ 3 Polytechnic Institute of NYU, USA. mdulieu@gmail.com \\ 4 Instituto de Matemáticas, Universidad Nacional Autónoma de México. \\ ruy@ciencias . unam.mx \\ 5 Stony Brook University, Stony Brook, NY 11794, USA. vi@vihart.com \\ 6 Universitat Politècnica de Catalunya, Jordi Girona 1-3, E-08034 Barcelona, Spain. \\ \{ferran.hurtado,maria.saumell, carlos.seara\}@upc.edu. Partially supported by \\ projects MTM2009-07242 and Gen. Cat. DGR 2009SGR1040.
}

\begin{abstract}
Given an ordered set of points and an ordered set of geometric objects in the plane, we are interested in finding a non-crossing matching between point-object pairs. We show that when the objects we match the points to are finite point sets, the problem is NP-complete in general, and polynomial when the objects are on a line or when their number is at most 2 . When the objects are line segments, we show that the problem is NP-complete in general, and polynomial when the segments form a convex polygon or are all on a line. Finally, for objects that are straight lines, we show that the problem of finding a min-max non-crossing matching is NP-complete.
\end{abstract}

\section{Introduction}

Finding a matching between pairs of planar objects, that is connecting them by a set of non-crossing line segments, is a natural problem that has been frequently studied in computational geometry. It is well known, for instance, that given two sets of $n$ points in the plane, say $n$ red points and $n$ blue points, there always exists a non-crossing matching between red and blue points. In particular, it is not difficult to show that the minimum Euclidean length matching is noncrossing. Kaneko and Kano [22] survey a number of related results. Algorithms for finding minimum sum and minimum bottleneck distance red-blue matchings are given in $[15,27]$.

In this paper, we investigate related questions for general planar objects instead of points. Again, matchings are represented by line segments, but here

\footnotetext{
* Chargé de Recherches du FRS-FNRS.
}

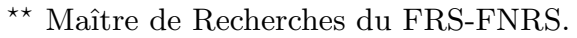


the endpoints can be placed anywhere inside the corresponding matched objects. Note that as a consequence of the aforementioned result on points, there always exists a non-crossing matching between two sets of objects. Here we consider the problem where we are given object pairs (i.e. a point and the geometric object it must be matched to) and need to find a set of non-crossing matching edges, if one exists. This can be seen as a 1-regular graph drawing problem with constraints on the location of vertices.

Related work. Problems on matchings have an important role in combinatorial graph theory, both for theoretical and applied aspects; hence a lot of research is devoted to the study of these problems (for example, see [24]).

Suppose we are given an embedding of a graph in the Euclidean plane, where the vertices are points in the plane, edges are rectilinear line segments, and weights on these edges represent the Euclidean distance between the vertices they connect. Elementary geometry tells us that the sum of any pair of opposite sides of a convex quadrilateral is strictly smaller than the sum of the diagonals. Remarkably, this implies that the minimum weight matching in any realization of the complete graphs $K_{2 n}$ and $K_{n, n}$ consists of pairwise non-crossing segments. These geometric graph problems can be solved using generic algorithms for weighted graphs. However, in the planar case just mentioned, Vaidya [27] proved that it is possible to obtain specialized algo-

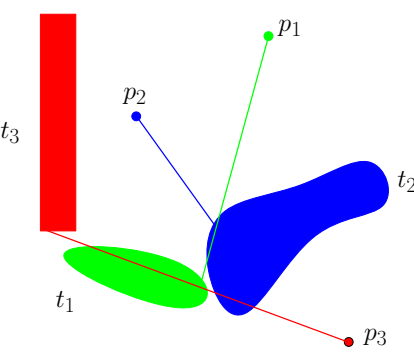

Fig. 1. A non-crossing matching for a set $P=\left\{p_{1}, p_{2}, p_{3}\right\}$ of points and a set $T=\left\{t_{1}, t_{2}, t_{3}\right\}$ of planar objects. rithms with better running times (the title of his paper is especially suggestive: Geometry helps in matching). In particular, in [27] the running time of the generic algorithm for the bipartite case was reduced from $O\left(n^{3}\right)$ to $O\left(n^{2.5} \log n\right)$. This was later improved to $O\left(n^{2+\varepsilon}\right)$ by Agarwal et al. [1]. Similar results have been obtained for other matching variations, such as bottleneck matching or uniform matching, in the work of Efrat, Itai and Katz [15]. The authors consider matchings as an approach for the problem of matching a point set $A$ with a point set $B$, where $A$ must be moved in some way to coincide as much as possible with $B$ or one of its subsets. This is a fundamental problem in pattern recognition [5, $7,8,10-12,19-21]$

The non-crossing requirement in our problems is quite natural in geometric scenarios (see for example $[25,2,3]$ ), and the family of geometric problems that we consider has several applications; these applications include geometric shape matching $[4,13,17,18]$, colour-based image retrieval [13], music score matching [26], and computational biology $[14,16]$.

Our results. Throughout the paper, we let $P:=\left\{p_{1}, p_{2}, \ldots, p_{n}\right\}$ be a set of points in the plane and $T:=\left\{t_{1}, t_{2}, \ldots, t_{n}\right\}$ be a set of planar objects. A matching for a pair $(P, T)$ consists of a set of line segments, called edges, of the form 
$\left\{p_{1} m_{1}, p_{2} m_{2}, \ldots, p_{n} m_{n}\right\}$, where $m_{i} \in t_{i}$. A matching is said to be non-crossing if no pair of matching edges properly cross. This is illustrated in Figure 1.

We consider the problem of deciding whether a non-crossing matching exists for a given pair $(P, T)$. In cases where a non-crossing matching always exists, we consider the problem of finding the matching that minimizes either the length of the longest edge, or the sum of the lengths of all the edges.

In Section 2, we study the case where the objects $t_{i}$ are finite point sets. We prove that the decision problem is NP-complete in general, but becomes polynomial when every $t_{i}$ has size at most two, or when all the $t_{i} \mathrm{~s}$ are on a line. In Section 3 we consider $T$ to be a set of line segments and prove that the $(P, T)$ matching problem is NP-complete. We also consider special cases, such as the case when the line segments form a convex polygon surrounding all points in $P$ (Section 4), or the case when segments belong to a single line (Section 5). We show that these special cases have polynomial solutions. Finally, in Section 6, we consider the problem of matching points with lines. In this variation, a noncrossing matching always exists, but the optimization problems are NP-hard.

\section{Matching Points with Finite Point Sets}

We first prove that if the objects $t_{i}$ are pairs of points, then we can decide whether there exists a non-crossing matching in polynomial time. On the other hand, if the sets $t_{i}$ may contain three points or more, the problem becomes NP-complete. This situation is similar to that of the $k$-satisfiability problem $(k-$ SAT). In $k$-SAT we are given a boolean formula $f$ of the form $C_{1} \wedge C_{2} \wedge \cdots \wedge C_{m}$ (where each $C_{i}$ is an OR clause of $k$ variables), and we are required to find a truth assignment of its variables that satisfy the formula. It is well-known that 2-SAT has a polynomial-time solution whereas $k$-SAT is NP-complete for $k \geq 3$. The 2-SAT problem can be solved in polynomial time by exploiting the fact that, if in a clause a variable is set to false, it forces the other variable to be set to true. This dependency between the variables can be represented by an implication graph.

An implication graph for the formula $f$ is a directed graph having two vertices for each variable $x_{i}$ of $f$, one of these vertices is labeled $x_{i}$ while the other is labeled $\neg x_{i}$. The vertex $x_{i}$ represents setting $x_{i}$ to true while $\neg x_{i}$ represents setting $x_{i}$ to false. Dependencies between literals in $f$ are represented by directed edges. Thus if $\left(x_{i} \vee \neg x_{j}\right)$ is a clause in $f$, in the implication graph there would be a directed edge from $\neg x_{i}$ to $\neg x_{j}$ and a directed edge from $x_{j}$ to $x_{i}$. These edges represent the fact that if $x_{i}$ is set to false then $x_{j}$ must also be set to false in order for the formula to be satisfied. Likewise if $x_{j}$ is set to true then $x_{i}$ must be set to true.

There exists a truth assignment satisfying $f$ if and only if no strong component of the implication graph contains both a vertex and its negation. The implication graph can be constructed in $O(m)$ time, where $m$ is the number of clauses. The previous condition can be verified in $O(m)$ time, and in general the strong components of a directed graph of $v$ vertices and $e$ edges can be computed 
in $O(e+v)$ time. A similar implication graph can be constructed for our problem when $t_{i}$ is a pair of points. Using this graph, it is possible to decide in $O\left(n^{2}\right)$ time whether $(P, T)$ has a non-crossing perfect matching.

Theorem 1. Given an ordered set $P$ of points and an ordered set $T$ of pairs of points, there is an algorithm that decides in $O\left(n^{2}\right)$ time whether $(P, T)$ has a non-crossing matching.

Proof. Assume that the elements of each $t_{i}$ are labeled arbitrarily " $\mathcal{T}_{i}$ " and " $\mathcal{F}_{i}$ " (thus $t_{i}=\left\{\mathcal{T}_{i}, \mathcal{F}_{i}\right\}$ ). We think of each $p_{i}$ as a boolean variable, so that if we match $p_{i}$ with $\mathcal{T}_{i}$ then $p_{i}$ is set to "true", and if $p_{i}$ is matched with $\mathcal{F}_{i}$, it is set to "false". We construct a directed implication graph $G$ as follows: For each $p_{i}$ we have vertices $p_{i, \mathcal{T}_{i}}$ and $p_{i, \mathcal{F}_{i}}$ in $G$. For every $i, j=1,2, \ldots, n$, we add the edge $\left(p_{i, X}, p_{j, Y}\right)$ ( $X$ equal to $\mathcal{T}_{i}$ or $\mathcal{F}_{i}$, and $Y$ equal to $\mathcal{T}_{j}$ or $\left.\mathcal{F}_{j}\right)$ to $G$ if and only if the line segments $\overline{p_{i}, X}$ and $\overline{p_{j}, \neg Y}$ intersect. For example if $\overline{p_{i}, \mathcal{T}_{i}}$ intersects $\overline{p_{j}, \mathcal{F}_{j}}$, we add the edge $\left(p_{i, \mathcal{T}_{i}}, p_{j, \mathcal{T}_{j}}\right)$ to $G$ (since if $p_{i}$ is matched to $\mathcal{T}_{i}, p_{j}$ must be matched to $\mathcal{T}_{j}$ as well). So $(P, T)$ has a non-crossing complete matching if and only if for every $p_{i}, p_{i, \mathcal{T}_{i}}$ and $p_{j, \mathcal{F}_{j}}$ lie in different strong connected components of $G$. Since $G$ is constructed in $O\left(n^{2}\right)$ time and has $O\left(n^{2}\right)$ edges, the overall complexity of the algorithm is $O\left(n^{2}\right)$.

\subsection{Matching points with triples}

By a reduction from Planar 3-SAT, we can prove that the problem of matching points with triples is NP-complete, even when the points within each triple are horizontally collinear. Details are omitted due to space limitations.

Theorem 2. Given an ordered set $P$ of points and an ordered set $T$ of triples of points, it is NP-complete to decide whether $(P, T)$ has a non-crossing matching. The problem remains NP-complete even if each triple is horizontally collinear.

\subsection{Matching points with $k$-tuples on a line}

Theorem 3. Given an ordered set $P$ of points and an ordered set $T$ of $k$-tuples of points on a line, we can decide in $O\left(k^{3} n^{2}\right)$ time whether $(P, T)$ has a noncrossing matching.

Proof. Without loss of generality, assume all the tuples are on a horizontal line $L$. Assume also that all points are on one side of $L$; otherwise we may consider each problem separately as the matching edges on each side of $L$ do not interact. We now show how to build a dynamic programming table that solves the problem.

In any solution to the problem, if a matching edge $e$ is part of the solution, then there is no matching edge that intersects $e$. Therefore, we can consider the regions on each side of $e$ (sub-problems) separately and determine whether they in turn have a valid solution. Thus, a sub-problem $\left(P^{\prime}, T^{\prime}\right)$ is defined as follows (see Figure 2): given a simple quadrilateral $A$ with one face adjacent to $L$, we want to decide if it is possible to find a non-crossing matching completely 


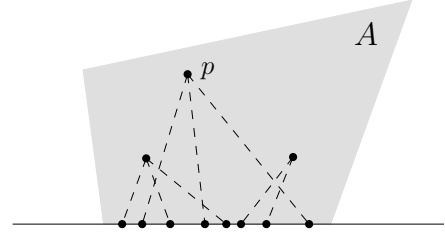

Fig. 2. Definition of a sub-problem.
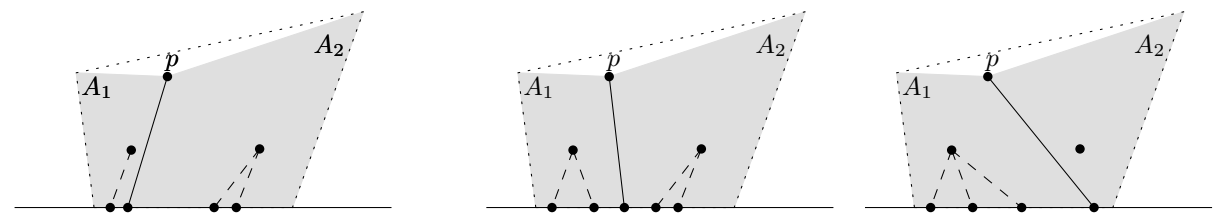

Fig. 3. In this example, there are three pairs of sub-problems to consider to decide if $p$ can be matched.

contained in the region $A$ for all the points contained in $A$, i.e., we want to solve the problem with $P^{\prime}=P \cap A$ and $T^{\prime}$ containing the subsets of the tuples of $T$ contained in $A$. If $A$ does not contain at least one point of $P$ (sub-problem of size 0 ), it is trivially true that there is a non-crossing matching. Otherwise, to solve the sub-problem we consider the topmost point $p$ in $A$. It has at most $k$ possible matching edges. If it has no possible matching edge, i.e., if all points that $p$ could be matched to in $T$ are out of $A$, then there is no valid matching.

Each of the possible matching edges defines two new independent sub-problems (see Figure 3 ) in the quadrilaterals $A_{1}$ and $A_{2}$, whose sizes are strictly smaller than that of the original problem, as there is one less point to match. To decide whether a matching exists for the original sets $P$ and $T$, we solve the sub-problem defined by the bounding box of both $P$ and $T$. Notice that all the sub-problems correspond to quadrilaterals defined by a pair of possible matching edges (or by the edges of the bounding box). Moreover, since in every sub-problem $A$ the $y$-coordinates of the corners of the bounding box are at least as large as that of every point in $A$, then the union of the regions of the sub-problems of $A$ will contain all the points in $A$. Thus no point will be left out.

The dynamic programming table has $k n+2$ rows and $k n+2$ columns, each of which corresponds to a possible matching edge or one of the left and right edges of the bounding box; the cells correspond to sub-problems (a pair of non-adjacent edges defines a quadrilateral), and we fill them with true or false depending on whether or not a matching exists for the considered sub-problem. Filling a cell of the table corresponds to solving at most $k$ pairs of sub-problems, which implies at most $2 k$ lookups in the table for each of the $O\left(k^{2} n^{2}\right)$ cells. Therefore, the total time and space required to solve the problem is $O\left(k^{3} n^{2}\right)$. 
The additional restriction of having points on a line greatly simplifies the problem, because the problem is NP-hard in the general case, but is polynomial for points on a line.

\section{Matching Points with Line Segments: General Case}

In this section we show that deciding the existence of a non-crossing matching between a set of points and a set of line segments is NP-complete, even if the segments are all horizontal or all have equal length. The proof uses appropriate gadgets to show that this problem reduces from the problem of matching points to triples (Theorem 2). It is omitted due to space limitations.

Theorem 4. Given an ordered set $P$ of points and an ordered set $T$ of line segments, it is NP-complete to decide whether $(P, T)$ has a non-crossing matching. The problem remains NP-complete even if all line segments in $T$ are horizontal or all have equal length.

\section{Matching Points to an Enclosing Convex Polygon}

In this special case of matching points with line segments, we assume the segments are the edges of a convex polygon and the points to be matched are inside the polygon, in general position.

We first describe some geometric properties of the input of this problem. We then describe an algorithm that runs in $O\left(n \log ^{2} n\right)$ time, and finds a non-crossing matching (if one exists) between a given set of point-segment pairs where the line segments form a convex polygon enclosing the points. This algorithm allows a minimum-length matching to be extracted easily.

Let $D^{o}=\left\{\Delta_{1}^{o}, \Delta_{2}^{o}, \ldots, \Delta_{n}^{o}\right\}$ be a set of triangles where each $\Delta_{i}^{o}$ is the triangle with apex $p_{i}$ and base $t_{i}$. Any valid matching edge $e_{i}$ must lie completely inside $\Delta_{i}^{o}$. Depending on the positions of other triangles in $D^{o}$, some candidate positions for $e_{i}$ can be identified as invalid because they would always cross other matching edges. By identifying such cases, triangle $\Delta_{i}^{o}$ can be reduced to a smaller triangle $\Delta_{i}$. At any time, the reduced triangle $\Delta_{i}$ has apex $p_{i}$ but its opposite base is a subsegment of $t_{i}$. Initially, $\Delta_{i}=\Delta_{i}^{o}$.

There are four ways in which two triangles $\Delta_{i}$ and $\Delta_{j}$ interact. The second case leads to a reduction rule. We describe the four cases below (see Figure 4):

1. $\Delta_{i}, \Delta_{j}$ are disjoint. In this case there will never be a direct interaction between the two.

2. $p_{j}$ is in $\Delta_{i}$, but $p_{i}$ is not in $\Delta_{j}$. In this case $\Delta_{i}$ should be reduced so that the two triangles become tangent (so that $p_{j}$ is no longer in $\Delta_{i}$ ).

3. $p_{i}$ is in $\Delta_{i}$ and $p_{j}$ is in $\Delta_{j}$. We call $\Delta_{i}$ and $\Delta_{j}$ inverted triangles, and cannot immediately make a reduction.

4. Both edges incident to each of $p_{i}$ and $p_{j}$ pairwise intersect. Then no noncrossing matching exists. 

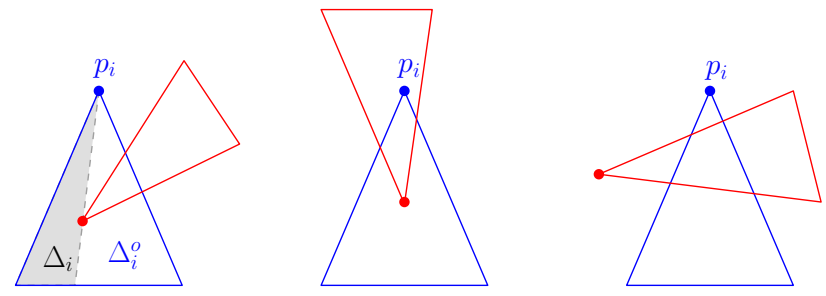

Fig. 4. Left: $\Delta_{i}^{o}$ is reduced to $\Delta_{i}$ (case 2). Middle: one of the two combinatorially distinct solutions (case 3). Right: no solution exists (case 4).

Note that in case (2) there is no choice but to reduce. The matching edge $e_{j}$ that is finally chosen will block any candidate $e_{i}$ that is outside the newly reduced $\Delta_{i}$. In case (3) there are two combinatorially valid placements for $e_{i}, e_{j}$, with respect to the positions of $p_{i}, p_{j}$. There is no reason to choose arbitrarily before verifying that neither triangle will be reduced further.

If we exhaustively apply our reduction rule to the triangles based on the cases described above, we would end up with a set having certain properties. Due to lack of space, we omit a detailed discussion of these properties.

Let two (three) mutually inverted triangles be called an inverted pair (triple). Let a unit be a (possibly reduced) triangle, an inverted pair, or an inverted triple.

Theorem 5. Given an ordered set $P$ of points inside a convex polygon having an ordered set $T$ of line segments as edges, deciding whether $(P, T)$ admits a non-crossing matching can be done in $O\left(n \log ^{2} n\right)$ time.

Proof. We provide an algorithm where we employ a divide-and-conquer technique. Suppose that we have solved the problem separately on two consecutive convex chains (we can transform a chain into a polygon by adding 3 fake edges and points; thus, solving the problem on a chain is equivalent to solving the polygonal version).

We claim that we can merge the two solutions in $O(n \log n)$ time. Each solution is a set of disjoint triangles and inverted pairs or triples. Refer to Figure 5.

Let $A$ and $B$ be two solved sub-problems of size $k$. We construct a standard point-location data structure on each in $O(k)$ time [23] by first triangulating $A$ and $B$ using Chazelle's linear-time triangulation algorithm [9]. Now, for every point $p_{i}$ in $B$, we locate $p_{i}$ in $A$ to determine if it is inside a unit in $A$. Note that $p_{i}$ can be in at most one unit. If it is, we determine if $\Delta_{i}$ reduces this unit by case (2). Likewise, for every point $p_{j}$ in $A$, we locate $p_{j}$ in $B$ to determine if it is inside a unit in $B$ and apply the appropriate reductions. Note that if at some moment $\Delta_{i}$ (belonging to $B$ ) gets reduced, this will not affect its corresponding unit in $A$; the same holds for all $\Delta_{j}$ in $A$ that get reduced.

Of course, it is possible that $\Delta_{i}$ will be inverted with a triangle in $A$. In this case we simply determine if there are reductions and, if applicable, we merge the two units. Therefore a constant number of reductions are applied per point, which means we spend $O(\log k)$ time per point for the point-location step. 
The only unresolved issue is to detect if case (4) will occur between triangles of $A$ and $B$ (see Figure 5-right). Given that all triangles have been reduced and merged into units, essentially we are verifying that no segments intersect. For this we can use the Bentley-Ottmann line segment intersection algorithm and stop as soon as a bad intersection is found [6]. For $k$ segments, such queries take $O(\log k+h \log k)$ time, where $h$ is the number of intersections reported. As we stop as soon as we report one intersection, $h=1$ and hence the total time is $O(\log k)$ per point. Thus our merge procedure takes $O(k \log k)$ time. By a simple recurrence analysis, we determine that the entire algorithm takes $O\left(n \log ^{2} n\right)$ time.

The algorithm described in the proof of Theorem 5 either decides that no solution exists, or otherwise produces a final set of reduced triangles that represents all valid solutions to the problem. In the latter case, every resulting unit is disjoint and thus independent of all others. So in each triangle we can easily pick the shortest joining segment, and in each inverted pair/triple, we try out the two possible choices and take the best matching. Therefore, after the algorithm finds a solution, the min-max and min-sum optimization problems can be solved in linear time.

\section{Matching Points with Segments on a Line}

As another special case of matching points to line segments, we now consider the case when the input line segments belong to one single line $L$. Throughout this section we will assume, without loss of generality, that $L$ is horizontal. As no matching edge will cross over $L$, our problem is split into two disjoint subproblems, and we focus on points above $L$. We consider two cases, depending on whether the segments are disjoint or not.

\subsection{Matching points with disjoint segments on a line}

Theorem 6. Given an ordered set $P$ of points above a horizontal line $L$ and an ordered set $T$ of disjoint line segments belonging to $L$, deciding whether $(P, T)$
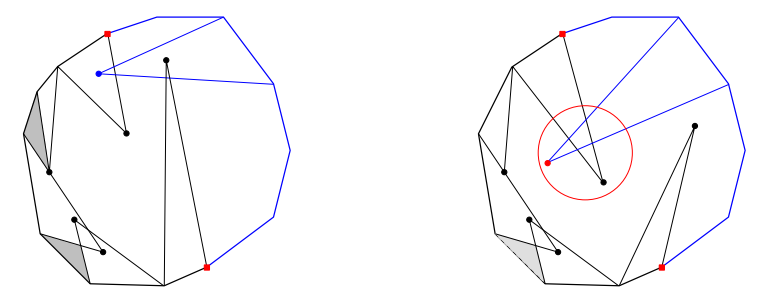

Fig. 5. Merging two solved sub-problems. In the left diagram, the grey regions in the left (black) sub-problem cannot contain points from the right (blue) sub-problem if there is a valid solution. In the right diagram, we see the type of event that we must check for after some initial reductions. 

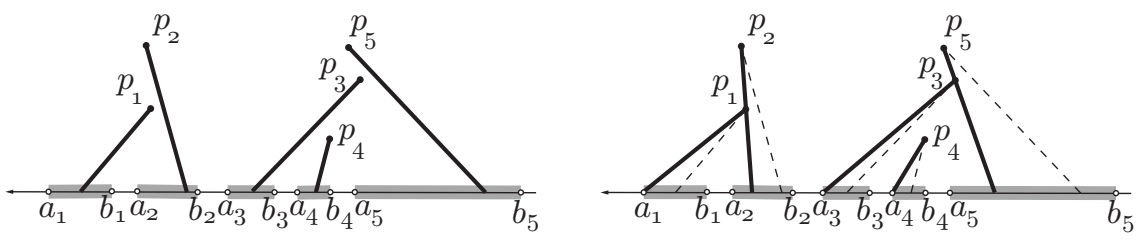

Fig. 6. Leftmost non-crossing matching (right) obtained from an initial non-crossing matching (left).

admits a non-crossing matching can be done in linear time. In the affirmative, the matching that minimizes the sum of the lengths of the edges can be found within the same time bound.

Proof. We denote by $\left[a_{i}, b_{i}\right]$ the interval corresponding to segment $t_{i}$, for $i=$ $1, \ldots, n$. Since the intervals are given in sorted order, we have $a_{1} \leq b_{1}<a_{2} \leq$ $b_{2}<\ldots<a_{n} \leq b_{n}$.

If $(P, T)$ admits some non-crossing matching $\left\{p_{1} m_{1}, p_{2} m_{2}, \ldots, p_{n} m_{n}\right\}$, where $a_{i} \leq m_{i} \leq b_{i}$ for all $i=1,2, \ldots, n$, we can always slide the point $m_{i}$ inside $t_{i}$ to a position $m_{i}^{L}$ as far to the left as possible (see Figure 6). This gives the unique leftmost non-crossing matching for $(P, T),\left\{p_{1} m_{1}^{L}, p_{2} m_{2}^{L}, \ldots, p_{n} m_{n}^{L}\right\}$. Notice that either $m_{i}^{L}=a_{i}$, or $p_{i}$ and $m_{i}^{L}$ are collinear with some $p_{j}$ with $j<i$.

Next we describe an algorithm for finding the leftmost non-crossing matching, if it exists. The algorithm considers points in a sequential greedy fashion, in the left-to-right order of the corresponding segments.

For $p_{1}$, the leftmost matching is simply given by the segment $p_{1} a_{1}$. We then consider the rays from the endpoints of this segment in the direction of the negative semiaxis of abscissae; their points at infinity can be symbolically described as $q_{0}=(-\infty, 0)$ and $q_{1}=\left(-\infty, y\left(p_{1}\right)\right)$.

The forbidden region is the (unbounded) region enclosed by an alternating sequence of horizontal line segments and subsegments of matched edges (See Figure 7). This region is updated at every step of the algorithm. Initially, it is described clockwise by its vertices, namely $q_{1} p_{1} a_{1} q_{0}$. Observe that if $p_{2}$ is inside the forbidden region, then a non-crossing matching $(P, T)$ would be impossible. If $p_{2}$ is outside the forbidden region, a matching is possible if and only if there is some point $m_{2}$ in the interval $a_{2} b_{2}$ such that the segment $p_{2} m_{2}$ does not cross the forbidden region. In the affirmative, we slide $m_{2}$ to its leftmost possible position, and shoot a ray from $p_{2}$ in the direction of the negative semiaxis of abscissae, which may go to infinity, or stop by hitting the segment $p_{1} a_{1}$. The forbidden region is updated in each case, and is always defined by alternating horizontal edges with portions of segments from the matching.

Assume that, in a generic step, we have obtained the leftmost matching $\left\{p_{1} m_{1}^{L}, p_{2} m_{2}^{L}, \ldots, p_{j-1} m_{j-1}^{L}\right\}$ and we are processing $p_{j}$. Let $q_{i_{1}} p_{i_{1}} q_{i_{2}} p_{i_{2}} \ldots q_{i_{k}} p_{i_{k}}$ $m_{i_{k}}^{L} q_{0}$ be the current forbidden region (refer to Figure 7). Observe that if there is some $m_{j} \in\left[a_{j}, b_{j}\right]$ such that the segment $p_{j} m_{j}$ can be added to the edges 


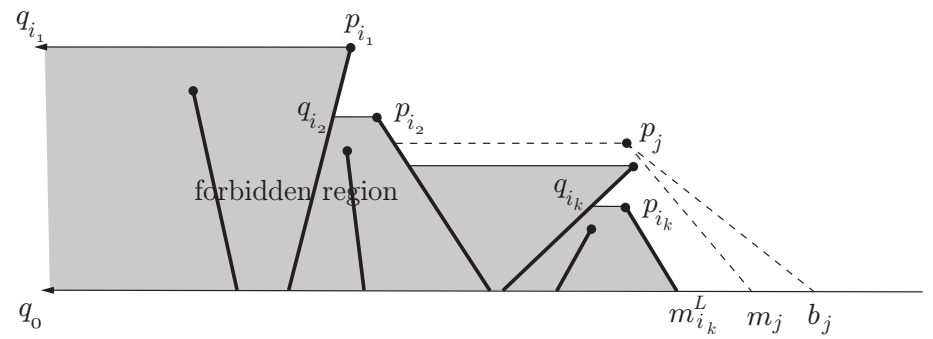

Fig. 7. Forbidden region and incremental step.
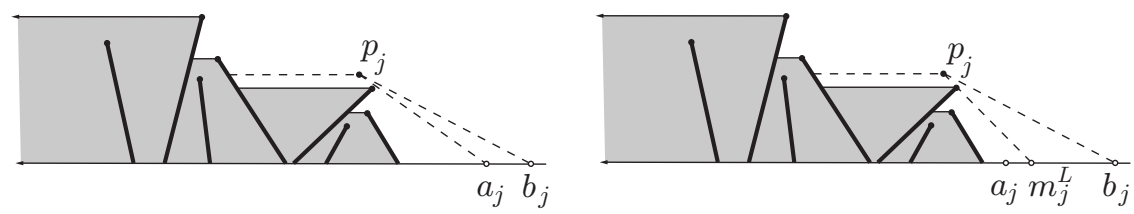

Fig. 8. Moving the new edge to the leftmost position.

found so far, getting a non-crossing matching, the segment $p_{j} b_{j}$ is also valid. We show next how to check the validity of $p_{j} b_{j}$.

We first check the $y$ coordinates of the points $m_{i_{k}}, p_{i_{k}}, p_{i_{k-1}} \ldots$, which form an increasing sequence, until we find that $y\left(p_{i_{t}}\right) \geq y\left(p_{j}\right) \geq y\left(p_{i_{t+1}}\right)$ (the case in which $y\left(p_{j}\right)$ is a maximum is completely analogous). Then, we check whether the segment $p_{j} b_{j}$ crosses the segments $m_{i_{k}} p_{i_{k}}, q_{i_{k-1}} p_{i_{k-1}}, \ldots, q_{i_{t-1}} p_{i_{t-1}}$. In the affirmative, the algorithm is over, as no crossing-free matching is possible. Otherwise, the segment $p_{j} b_{j}$ is valid. We slide the point matched with $p_{j}$ as much to the left as possible (Figure 8), which can be done by finding the angularly closest point among $p_{i_{t+1}}, p_{i_{t+2}}, \ldots, p_{i_{k}}, a_{j}$.

If we shoot a ray from $p_{j}$ in the direction of the negative semiaxis of abscissae, we hit the boundary of the forbidden region in a point $q_{j}$, possibly at infinity, and the forbidden region is updated to be $q_{i_{1}} p_{i_{1}} q_{i_{2}} p_{i_{2}} \ldots p_{i_{t}} q_{j} p_{j} m_{j}^{L} q_{0}$.

The cost of the step for $p_{j}$ is proportional to the size of the forbidden polygonal region that disappears, and that will never be processed again. Therefore, the amortized cost of one step is constant and the global cost of the algorithm is $O(n)$. At the end we obtain the leftmost matching $\left\{p_{1} m_{1}^{L}, p_{2} m_{2}^{L}, \ldots, p_{n} m_{n}^{L}\right\}$, unless no matching is possible.

If $(P, T)$ admits a non-crossing matching, with a symmetrical algorithm we can obtain the rightmost matching $\left\{p_{1} m_{1}^{R}, p_{2} m_{2}^{R}, \ldots, p_{n} m_{n}^{R}\right\}$. Then any points $m_{i}$ in the intervals $\left[m_{i}^{L}, m_{i}^{R}\right]$ provide a non-crossing matching $\left\{p_{1} m_{1}, p_{2} m_{2}, \ldots\right.$, $\left.p_{n} m_{n}\right\}$. In particular, in each interval $\left[m_{i}^{L}, m_{i}^{R}\right]$ we can pick the matching point $m_{i}$ which is closest to $p_{i}$, and hence obtain the matching that minimizes the sum of the lengths of the edges in additional $O(n)$ time. 


\subsection{Matching points with arbitrary segments on a line}

In this section, we show that when the given segments are confined to a line and possibly intersect, we can determine the existence of a non-crossing matching in polynomial time. The proof first discretizes the problem, and then uses the same approach as in the proof of Theorem 3 for $k$-tuples with $k=O\left(n^{2}\right)$. Details of the proof are omitted from this version of the paper.

Theorem 7. Given an ordered set $P$ of points above a horizontal line $L$ and an ordered set $T$ of line segments belonging to $L$, deciding whether $(P, T)$ admits a non-crossing matching can be done in $O\left(n^{8}\right)$ time.

\section{Matching Points with Lines}

In the case where points are matched with lines, it is easy to see that a noncrossing matching always exists: choose an arbitrary direction, not parallel to any line, and project each point on its corresponding line in that direction.

Here we consider the optimization problem of minimizing the maximum length over all matching edges. The proof, omitted here due to space limitations, uses a reduction from the problem of deciding the existence of a non-crossing matching between a set of points and a set of segments.

Theorem 8. Given an ordered set $P$ of points and an ordered set $T$ of lines, finding a min-max non-crossing matching of $(P, T)$ is NP-complete.

\section{References}

1. P. Agarwal, B. Aronov, M. Sharir, and S. Suri. Selecting distances in the plane. Algorithmica, 9(5):495-514, 1993.

2. O. Aichholzer, S. Bereg, A. Dumitrescu, A. Garca, C. Huemer, F. Hurtado, M. Kano, A. Mrquez, D. Rappaport, S. Smorodinsky, D. Souvaine, J. Urrutia, and D. R. Wood. Compatible geometric matchings. Computational Geometry: Theory and Applications, 42:617-626, 2009.

3. O. Aichholzer, S. Cabello, R. Fabila-Monroy, D. Flores-Penaloza, T. Hackl, C. Huemer, F. Hurtado, and D. Wood. Edge-Removal and Non-Crossing Configurations in Geometric Graphs. In Proceedings of 24th European Conference on Computational Geometry, pages 119-122, 2008.

4. H. Alt and L. Guibas. Discrete geometric shapes: Matching, interpolation, and approximation. Handbook of computational geometry, pages 121-154, 1999.

5. E. Arkin, K. Kedem, J. Mitchell, J. Sprinzak, and M. Werman. Matching points into noise regions: combinatorial bounds and algorithms. In Proceedings of the second annual ACM-SIAM symposium on Discrete algorithms, pages 42-51, 1991.

6. J. L. Bentley and T. A. Ottmann. Algorithms for reporting and counting geometric intersections. IEEE Transactions on Computers, 28(9):643-647, 1979.

7. S. Cabello, P. Giannopoulos, C. Knauer, and G. Rote. Matching point sets with respect to the Earth Mover's Distance. Computational Geometry: Theory and Applications, 39(2):118-133, 2008. 
8. D. Cardoze and L. Schulman. Pattern matching for spatial point sets. In Proceedings. 39th Annual Symposium on Foundations of Computer Science (FOCS), 1998, pages 156-165, 1998.

9. B. Chazelle. Triangulating a simple polygon in linear time. Discrete and Computational Geometry, 6(1):485-542, 1991.

10. L. Chew, D. Dor, A. Efrat, and K. Kedem. Geometric pattern matching in ddimensional space. Discrete and Computational Geometry, 21(2):257-274, 1999.

11. L. Chew, M. Goodrich, D. Huttenlocher, K. Kedem, J. Kleinberg, and D. Kravets. Geometric pattern matching under Euclidean motion. Computational Geometry: Theory and Applications, 7(1-2):113-124, 1997.

12. L. Chew and K. Kedem. Improvements on geometric pattern matching problems. In Proceedings of the Third Scandinavian Workshop on Algorithm Theory, pages 318-325. Springer, 1992.

13. S. Cohen. Finding color and shape patterns in images. PhD thesis, Stanford University, Department of Computer Science, 1999.

14. J. Colannino, M. Damian, F. Hurtado, J. Iacono, H. Meijer, S. Ramaswami, and G. Toussaint. An $O(n \log n)$-time algorithm for the restriction scaffold assignment problem. Journal of Computational Biology, 13(4):979-989, 2006.

15. A. Efrat, A. Itai, and M. Katz. Geometry helps in bottleneck matching and related problems. Algorithmica, 31(1):1-28, 2001.

16. A. Formella. Approximate point set match for partial protein structure alignment. Proceedings of Bioinformatics: Knowledge Discovery in Biology (BKDB2005). Facultade Ciencias Lisboa da Universidade de Lisboa, pages 53-57, 2005.

17. P. Giannopoulos and R. Veltkamp. A pseudo-metric for weighted point sets. In Proc. of the 7th European Conf. Comp. Vision, 2002.

18. K. Grauman and T. Darrell. Fast contour matching using approximate earth mover's distance. In Proceedings of the 2004 IEEE Computer Society Conference on Computer Vision and Pattern Recognition, pages 220-227, 2004.

19. P. Heffernan. Generalized approximate algorithms for point set congruence. In Proceedings of the 3rd Workshop on Algorithms and Data Structures, pages 373373, 1993.

20. P. Heffernan and S. Schirra. Approximate decision algorithms for point set congruence. In Proceedings of the eighth annual Symposium on Computational geometry, pages 93-101, 1992.

21. D. Huttenlocher and K. Kedem. Efficiently computing the Hausdorff distance for point sets under translation. In Proceedings of the Sixth ACM Symposium on Computational Geometry, pages 340-349, 1990.

22. A. Kaneko and M. Kano. Discrete geometry on red and blue points in the plane - a survey. Discrete \& Computational Geometry, 25:551-570, 2003.

23. D. Kirkpatrick. Optimal search in planar subdivisions. SIAM Journal on Computing, 12(1):28-35, 1983.

24. L. Lovász and M. D. Plummer. Matching theory. Elsevier Science Ltd, 1986.

25. D. Rappaport. Tight bounds for visibility matching of $f$-equal width objects. In Proceedings of the Japanese Conference on Discrete and Computational Geometry (JCDCG'02), pages 246-250, 2002.

26. R. Typke, P. Giannopoulos, R. Veltkamp, F. Wiering, and R. Van Oostrum. Using transportation distances for measuring melodic similarity. In Proceedings of the 4th International Conference on Music Information Retrieval (ISMIR 2003), pages 107-114, 2003.

27. P. Vaidya. Geometry helps in matching. In STOC '88: Proceedings of the twentieth annual ACM symposium on Theory of computing, pages 422-425. ACM, 1988. 